\title{
Stimmungsbarometer
}

\section{Ein Drittel der deutschen Ärzte ist unzufrieden}

Die Stimmung unter Deutschlands Ärzten ist nicht gut. Vor allem Niedergelassene klagen über schlechte Arbeitsbedingungen. Trotzdem will jeder Fünfte demnächst in die Praxis investieren. Generell beruflich verändern wollen sich allerdings nur wenige.

Z u viel Bürokratie, stagnierendes Einkommen, zu hohe Arbeitsbelastung und einengende Budgets - das sind nur einige Gründe, warum viele Ärzte in Deutschland längst nicht mehr über ihre berufliche Situation jubeln. Über ein Drittel der deutschen Ärzte gibt in einer aktuellen, repräsentativen Studie von TNS Infratest an, mit der derzeitigen Situation alles andere als zufrieden zu sein. Die Stimmung bei den Niedergelassenen ist besonders schlecht: Hier sind $44 \%$ unzufrieden, bei den angestellten Ärzten ist es fast jeder Fünfte.

Die Marktforscher hatten im Auftrag der Commerzbank 300 Ärzte befragt, darunter 140 niedergelassene und 160 angestellte Ärzte.

\section{Investitionen trotz schlechter Stimmung}

Überraschend ist, dass trotz der relativ schlechten Grundstimmung jeder fünfte niedergelassene Arzt in diesem Jahr eine größere Investition plant - nicht selten betrifft dies eine Kooperationsgründung. Das deckt sich zwar mit der Aussage, dass auch jeder fünfte Niedergelassene eine berufliche Veränderung im nächsten Jahr anstrebt, überraschend ist aber zugleich, dass über zwei Drittel die beruflichen Dinge - zumindest fürs nächste Jahr - eher so lassen wollen, wie sie sind.

Die berufliche Veränderung suchen im Laufe des nächsten Jahres vor allem angestellte Ärzte (57\%). Das mag auch daran liegen, dass die Befragten in den Angestelltenverhältnissen besonders unter den derzeitigen Arbeitsbedingungen wie Arbeitszeiten, -belastung und -klima leiden (64\%). Jeweils rund die Hälfte der nieder- gelassenen Ärzte nennt dagegen die Bürokratie und stagnierende oder sinkende Einnahmen als Gründe für die Unzufriedenheit. Etwas mehr als ein Viertel klagt zudem über zu enge Budgetvorgaben in der Patientenversorgung.

\section{Niederlassung angestrebt}

Die Angst, dass gerade angestellte Ärzte ins Ausland abwandern werden, bestätigt sich nicht unbedingt: Lediglich 29\% der angestellten Ärzte, die sich beruflich verändern wollen, sehen im Ausland eine Option. Das Gros, nämlich $44 \%$, will sich niederlassen. Sie planen entweder eine Praxisgründung oder -übernahme (18\%), wollen eine Kooperation eingehen (18\%) oder als Mitunternehmer in ein MVZ einsteigen.

Allerdings zieht es die niederlassungswilligen angestellten Ärzte eher in die Stadt als aufs Land: Gerade einmal $4 \%$ der angestellten Ärzte und nur $5 \%$ beider befragten Gruppen würde sich auf dem Land niederlassen, wobei unter den bereits niedergelassenen Medizinern immerhin $9 \%$ die ländlichen Regionen bei künftigen beruflichen Veränderungen bevorzugen würden. Für die Großstadt interessieren sich $40 \%$ der angestellten Ärzte und $42 \%$ beider Gruppen, in eine Klein-/oder Mittelstadt würden immerhin noch $38 \%$ der angestellten und über ein Drittel aller veränderungswilligen Ärzte ziehen.

\section{Nachwuchs will nicht aufs Land}

Das geringe Interese an den ländlichen Regionen sieht die Kassenärztliche Bundesvereinigung (KBV) besonders kritisch, denn bereits eine bundesweite Befragung von Medizinstudenten im vergangenen
Stimmungstief bei Deutschlands Ärzten?

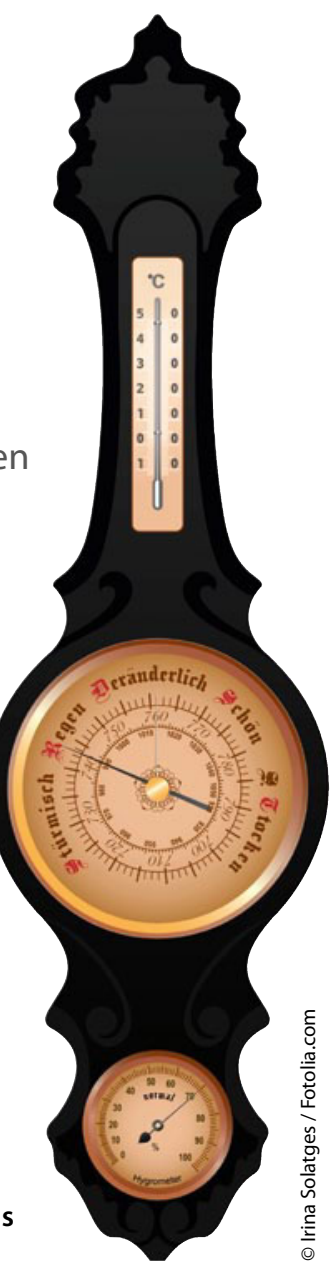

Sommer ergab, dass für die große Mehrheit der Mediziner eine Tätigkeit als Landarzt nicht infrage kommt. Das sogenannte Berufsmonitoring war eine gemeinsame Studie der KBV mit der Universität Trier. Von den 12.500 befragten Studenten konnten sich $16 \%$ eine Tätigkeit als Landarzt vorstellen, heißt es in einer Mitteilung der KBV. „Damit bestätigt die aktuelle Umfrage unsere Ergebnisse“, erläutert KBV-Vorstand Dr. Carl-Heinz Müller. Dabei kamen Städte mit bis zu 5.000 Einwohnern für rund die Hälfte der befragten Studenten auf keinen Fall als Arbeitsort infrage. „Beide Umfragen zeigen, dass dringend etwas geschehen muss, um dem Ärztemangel insbesondere auf dem Land noch etwas entgegenzusetzen“, so Müller. Dazu gehöre neben Bürokratieabbau und einer angemessenen Vergütung auch eine entsprechende Infrastruktur, die auch für die Partner und Familien der Ärzte Anreize schaffe. „Sonst ist der Landarzt bald wirklich nur noch im Fernsehen zu sehen", meint Müller.

reh 